\title{
PESQUIMAT
}

Revista del Instituto de

Investigación de la Facultad

de Ciencias Matemáticas de la U.N.M.S.M

Vol. N'I.pp 01.26 Lima - Perú Julio-1998

\section{HIDDEN REGULARITY FOR A STRONGLY NON LINEAR}

\section{WAVE EQUATION}

\author{
JAIME E. MUÑOZ RIVERA ${ }^{1}$
}

LNCC - Rio de Janeiro - Brazil

\section{ABSTRACT}

In this paper we consider the nonlinear wave equation,

$$
\begin{cases}u^{\prime \prime}-\Delta u+f(u)=V & \text { in } Q=\Omega \mathrm{x}] 0, T[ \\ u(0)=u_{0}, u^{\prime}(0)=u_{1} & \text { in } \Omega, \\ u(x, t)=0 & \text { on } \left.\sum=\Gamma \times\right] 0, T[\end{cases}
$$

where $f$ is a continuous function satisfying

$$
\limsup _{|S| \rightarrow+\infty} \frac{f(s)}{s}>-\infty
$$

and $\Omega$ is a bounded domain of $\mathbb{R}^{n}$ with smooth boundary $\Gamma$. We prove that there exist a solution for $\left({ }^{*}\right)$ that satisfies the regularity conditions: $\frac{\partial u}{\partial \eta} \in L^{2}\left(\sum\right)$.

\footnotetext{
${ }^{1}$ Investigador del Laboratorio Nacional de Computación Científica (LNCC) de Rio de Janeiro - Brazil
} 
Moreover we have that there exist a constant $\mathcal{C}>0$ such that,

$$
\left|\frac{\partial u}{\partial \eta}\right| \leq \mathcal{C}\left\{E(0)+|V|_{Q}^{2}\right\}
$$

\section{INTRODUCTION}

Let $\Omega$ be an open bounded set of $\mathbb{R}^{n}$, with boundary $\Gamma$ of class $\mathcal{C}^{2}$. Set $\left.Q=\Omega \times\right] 0, T[$ and $\left.\sum=\Gamma x\right] 0, T\left[\right.$. We will denote by $(\cdot, \cdot)_{\Omega}$ and $(\cdot, \cdot)_{Q}$ the inner product of $L^{2}(\Omega)$ and $L^{2}(Q)$ respectively and by $|.|_{\Omega},||_{Q}$ and $\|$.$\| , the norms in L^{2}(\Omega), L^{2}(Q)$, and $H_{0}^{1}(\Omega)$ respectively. We consider the nonliner problem:

$$
\begin{cases}u^{\prime \prime}-\Delta u+f(u)=V & \text { in } Q \\ u(0)=u_{0}, u^{\prime}(0)=u_{1} & \text { in } \Omega \\ u(x, t)=0 & \text { on } \Sigma\end{cases}
$$

In J.L.Lions [1] was study the hidden regularity for system (1.1) when $f(s)=$ $s^{3}$ and more generality for a $f(s)=s|s|^{e}$, where $e \geq 0$. In this work we are going to study the hidden regularity for the solution of the problem (1.1) when $f$ is a 
continuous function satisfying,

$$
\limsup _{|S| \rightarrow+\infty} \frac{f(s)}{s}>-\infty
$$

That is, we will show that there exist a solution $u$ of the above problem such that the normal derivate of $u$ belongs to $L^{2}(\Sigma)$. Moreover we will prove that there exist a constant $\mathcal{C}>0$ such that:

$$
\left|\frac{\partial u}{\partial \eta}\right|_{\Sigma} \leq C E_{0}
$$

where $E_{0}$ is the initial energy of the system (1.1).

$$
\left(E_{0}=\frac{1}{2}\left|u_{1}\right|_{\Omega}^{2}+\int_{\Omega} G\left(u_{0}\right) d x\right)
$$

where $G(s)=\int_{0}^{s} f(\eta) d \eta$. 


\section{EXISTENCE AND HIDDEN REGULARITY}

First of all we are going to construct a sequence of real numbers $\left(s_{\nu}\right)_{\nu \in \mathbb{N}}$ and $\left(s_{-\nu}\right)_{\nu \in \mathbb{N}}$ satisfying the following conditions,

$$
\begin{aligned}
& s_{\nu} \geq \nu, \forall \nu \in \mathbb{N},\left|f\left(s_{\nu}\right)\right| \leq \mathcal{C}+|f(s)|, \forall s \geq \nu \\
& s_{-\nu} \geq-\nu, \forall \nu \in \mathbb{N},\left|f\left(s_{\nu}\right)\right| \leq \mathcal{C}+|f(s)|, \forall s \geq-\nu
\end{aligned}
$$

This sequences are going to play and important role in the sequel.

LEMMA 2.1.- Let's $f$ be a continuous function defined in $\mathbb{R}$, then there exists a sequence of real numbers, $\left(s_{\nu}\right)_{\nu \in \mathbb{N}}$, and a positive constant $\mathcal{C}$, independing of $\nu_{1}$ satisfying condition (2.1) and (2.2).

PROOF.- Let's consider the following problem.

$$
I_{\nu}=\operatorname{in} f\{|f(s)| ; s \geq \nu\}
$$

If for all $\nu \in \mathbb{N}$, there exist $s \nu \geq \nu$ such that $f\left(s_{\nu}\right)=I_{\nu}$, then this sequence satisfies condition (2.1). Now we can suppose that there exist a $v_{0}$ such that,

$$
f(s)>I_{V_{0}} \text { for all } s \geq v_{0}
$$


This relations emply that $I_{\nu}=I_{\nu_{0}}$ for all $\nu \geq v_{0}$. Let us put. $I_{0}=I \nu_{0}$. Since $I_{0}=\inf |s(s)| ; s \geq V_{0}$, there exists a sequence $\left(t_{k}\right)_{k \in \mathbb{N}}$ such that,

$$
f\left(\bar{t}_{k}\right) \rightarrow I_{0}
$$

from the continuity of $f$ we conclude that $t_{k}$ is not bounded, then there exist a subsequence $\left(t_{k_{\nu}}\right)_{\nu \in \mathbb{N}}$ satisfying:

$$
t_{k_{\nu}} \geq V, \quad \nu \in \mathbb{N}
$$

Let us put $s_{\nu}=t_{k_{\nu}}$, from (2.4) we obtain that there exist a constant $\mathcal{C}$ (independing of $\nu$ ) such that $\left|f\left(s_{\nu}\right)\right|=\left|f\left(t_{k_{\nu}}\right)\right| \leq \mathcal{C}$, finally from (2.5) we conclude that $\left(s_{\nu}\right)_{\nu \in \mathbb{N}}$ satisfies condition (2.1). By the same arguments we can prove the existence of a sequence $\left(s_{\nu}\right)_{\nu \in \mathbb{N}}$ satisfying condition (2.2), only consider the problem

$$
I_{-\nu}=\inf |f(s)| ; s \leq-\nu,
$$

and the result follows.

With the sequences $\left(s_{\nu}\right)_{\nu \in \mathbb{N}}$ and $\left(s_{-\nu}\right)_{\nu \in \mathbb{N}}$ constructed in Lemma 2.1 we define 
a sequence $\left(f_{\nu}\right)_{\nu \in \mathbb{N}}$ of continuous function in the following way:

$$
f_{\nu}(s)= \begin{cases}f(s) & \text { if } \quad s_{\nu} \leq s \leq s_{\nu} \\ f\left(s_{\nu}\right) & \text { if } \quad s \geq s_{\nu} \\ f\left(s_{-\nu}\right) & \text { if } \quad s_{\nu} \leq s_{-\nu}\end{cases}
$$

As a consequence of Lemma 2.1 we have that the sequence $\left(f_{\nu}\right)_{\nu \in \mathbb{N}}$ satisfies the following properties:

$$
\left|f_{\nu}(s)\right| \leq \mathcal{C}+|f(s)| \quad \text { for all } \nu
$$

$$
f_{\nu} \rightarrow f \quad \text { uniformly on bounded sets }
$$

LEMMA 2.2.- Let's $f$ be a continuous function satisfying condition (1.2), and $\left(f_{\nu}\right)_{\nu \in \mathbb{N}}$, the sequence defined in (2.6). Then there exist a positive constant $\mathcal{C}_{0}$ such that.

$$
\begin{gathered}
s f_{\nu}(s) \geq-\mathcal{C}_{0}\left(s^{2}+1\right) \quad \forall s \in \quad \forall v \geq v_{0} \\
\int_{0}^{t} f_{\nu}(s) d s \geq-\mathcal{C}_{0}\left(t^{2}+2|t|\right)
\end{gathered}
$$




$$
\left|\int_{0}^{t} f_{\nu}(s) d s\right| \leq \frac{1}{2} \mathcal{C}_{0}|t(t+3)|+\int_{0}^{t} f(s) d s
$$

PROOF.- First of we are going to prove that there exists a positive constant $\mathcal{C}_{0}$, such that

$$
f(s) \geq-\mathcal{C}_{0} s \quad \forall s \geq N \quad \text { and } \quad f(s) \leq-\mathcal{C}_{0} s \quad \forall s \leq N
$$

In fact, if $\liminf s^{-1} f(s)=+\infty$ the expression (2.12) is valid. Now we can suppose that $\liminf s^{-1} f(s)=x<+\infty$, then for $\mathcal{E}>0$, there exist $N>0$ such that $s^{-1} f(s)>x^{-\mathcal{E}}$, for $|s| \geq N$. Let us take $\mathcal{C}=\sup \{|f(s)| ;|s| \leq N\}$, $\mathcal{C}_{2}=\sup \{|s f(s)| ;|s| \leq N\}$, and put $\mathcal{C}_{0}=\max \left\{\mathcal{C}, \mathcal{C}_{1}, \mathcal{C}_{2},|x-\mathcal{E}|\right\}$ where $\mathcal{C}$ is the constant in (2.7), certainly for this $\mathcal{C}_{0}$, condition (2.12) is valid. Finally multiplying the relations in (2.12) by $s(|s| \geq N)$, we have by the definition of $\mathcal{C}_{0}$, that the first part of (2.9) is valid. The second part of (2.9) follows from (2.1), (2.2), (2.6) and also, the definition of $\mathcal{C}_{0}$, for $v_{0}=N$.

In order to prove (2.11), let us note that from (2.12) follows that:

$$
f_{\nu}(s) \geq-\mathcal{C}_{0}(s+1) \quad \forall s \geq 0 \text { and } f_{\nu}(s) \leq-\mathcal{C}_{0}(s-1) \quad \forall s \leq 0
$$


Integrating this expression we obtain (2.10). In order to obtain (2.11) let us consider relation (2.7) then we have:

$$
\left|\int_{0}^{t} f_{\nu}(s) d s\right| \leq \int_{0}^{t}\left|f_{\nu}(s)\right| d s \mathcal{C}|t|+\int_{0}^{t}|f(s) d s| \quad \forall t \in \mathbb{R}
$$

From (2.12) we obtain that $f(s) \geq-\mathcal{C}_{0}(s+1) \quad \forall s \geq 0$, which imply that $|f(s)| \leq f(s)+2_{\mathcal{c}} 0(s+1) \quad \forall s \geq 0$. Then we have:

$$
\int_{0}^{t}|f(s) d s| \leq \int_{0}^{t} f(s) d s+\mathcal{C}_{0} t(t+2) \quad \forall t \geq 0
$$

and since $f(s) \leq|f(s)|$ we obtain,

$$
\int_{0}^{t}|f(s)| d s \leq \int_{0}^{t} f(s) d s \quad \forall t \geq 0
$$

Finally from (2.15) and (2.16) we obtain (2.11).

Let us denote by $G_{\nu}(t)=\int_{0}^{t} f_{\nu}(s) d s$, then we have that

$$
G_{\nu} \rightarrow G \quad \text { uniformly on bounded sets. }
$$

Before to prove the main result of this paper we will prove an identity that 
will be fundamental in that follows.

LEMMA 2.3.- Le $h$ be a contimuous function. Let $q=\left(q_{k}\right)$ a field of vectors of class $\left[\mathcal{C}^{1}(\bar{\Omega})\right]^{n}$. Then for all $W$ satisfying.

$$
\begin{gathered}
W \in L^{2}\left(0, T ; H_{0}^{1}(\Omega) \eta H^{2}(\Omega)\right), h(W) \in L^{1}(Q) \\
W^{\prime} \in L^{2}\left(0, T ; H_{0}^{1}(\Omega)\right)
\end{gathered}
$$

$$
W^{\prime \prime} \in L^{2}\left(0, T ; L^{2}(\Omega)\right)
$$

$$
\left\{\begin{array}{l}
W^{\prime \prime}-\Delta W+h(W)=V \quad \text { in } \quad Q \\
W(0)=W_{0}, W^{\prime}(0)=W_{1} \quad \text { in } \Omega \\
W(x, t)=0 \quad \text { on } \sum
\end{array}\right.
$$


Where $H$ is the primitive of $h$. Then we have.

$$
\begin{aligned}
& \frac{1}{2} \int_{\sum} q_{k} n_{k}\left|\frac{\partial W}{\partial \eta}\right|^{2} d \sum=\left[\left(W^{\prime}(t), q_{k} \frac{\partial W(t)}{\partial x_{k}}\right]_{0}^{T}+\right. \\
& +\int_{Q} \frac{\partial q_{k}}{\partial z_{j}}\left\{\left|W^{\prime}\right|^{2}-|\nabla W|^{2}-2 H(W)\right\} d x d t \\
& +\int_{Q} \frac{\partial q_{k}}{\partial z_{j}} \times \frac{\partial W}{\partial x_{k}} \times \frac{\partial W}{\partial x_{j}} d x d t-\int_{Q} V_{q_{k}} \frac{\partial W}{\partial x_{k}}
\end{aligned}
$$

PROOF.-Let us multiply (2.21), by $q_{k} \frac{\partial W}{\partial x_{k}}$, then we have that:

$$
\begin{gathered}
\int_{Q}\left\{W^{\prime \prime}-\Delta W+h(W)\right\} q_{k} \frac{\partial W}{\partial x_{k}} d x d t= \\
\int_{Q} V q_{k} \frac{\partial W}{\partial x_{k}} d x d t
\end{gathered}
$$

Let us denote by:

$$
\begin{gathered}
I_{1}=\int_{Q} W^{\|} q_{k} \frac{\partial W}{\partial x_{k}} d x d t \\
I_{2}=\int_{Q} \Delta W q_{k} \frac{\partial W}{\partial x_{k}}
\end{gathered}
$$

then we have:

$$
\begin{aligned}
I_{1} & =\left[\left(W^{\prime}(t), q_{k} \frac{\partial W}{\partial x_{k}}(t)\right)\right]_{0}^{T}-\int_{0} W^{\prime} q_{k} \frac{\partial W^{\prime}}{\partial x_{k}} d x d t \\
& =\left[\left(W^{\prime}(t), q_{k} \frac{\partial W(t)}{\partial x_{k}}\right)\right]_{0}^{T}-\frac{1}{2} \int_{0} q_{k} \frac{\partial\left|W^{\prime}\right|^{2}}{\partial x_{k}} d x d t
\end{aligned}
$$


from where we obtain that:

$$
I_{1}=\left[\left(W^{\prime}(t), q_{k} \frac{\partial W(t)}{\partial x_{k}}\right)_{\Omega}\right]_{0}^{T}+\frac{1}{2} \int_{0} \frac{\partial q_{k}}{\partial x_{k}}\left|W^{\prime}\right|^{2} d x d t
$$

on the other hand we have that:

$$
\begin{aligned}
I_{2} & =-\int_{Q} \frac{\partial W}{\partial x_{j}} \mathbf{x} \frac{\partial}{\partial x_{j}}\left\{q_{k} \frac{\partial W}{\partial x_{h}}\right\} d x d t+\int_{\sum} q_{k} \frac{\partial W}{\partial x_{h}} \times \frac{\partial W}{\partial \eta} d \sum \\
& =-\int_{Q} \frac{\partial W}{\partial x_{k}} \frac{\partial q_{k}}{\partial x_{k}} \times \frac{\partial W}{\partial x_{h}} d x d t-\int_{Q} \frac{\partial W}{\partial x_{j}} \times \frac{\partial^{2} W}{\partial x_{k} \partial x_{j}} \times q_{k} d x d t . \\
& +\int_{\sum} q_{k} \frac{\partial W}{\partial x_{h}} \times \frac{\partial W}{\partial \eta} d \sum . \\
& =-\int_{Q} \frac{\partial W}{\partial x_{k}} \times \frac{\partial q_{k}}{\partial W_{j}} \times \frac{\partial W}{\partial x_{k}} d x d t-\frac{1}{2} \int_{Q} q k \frac{\partial}{\partial x_{k}}|\nabla W|^{2} d x d t+\int_{\sum} q_{k} \frac{\partial W}{\partial x_{k}} \times \frac{\partial W}{\partial \eta} d \Sigma .
\end{aligned}
$$

But since $W=0$ on $\sum$ we have that:

$$
\frac{\partial W}{\partial x_{k}}=\eta_{k} \frac{\partial W}{\partial \eta} \text { on } \sum
$$

and

$$
|\nabla W|^{2}=\left|\frac{\partial W}{\partial \eta}\right|^{2} \text { on } \sum
$$


we have:

$$
\begin{aligned}
I_{2} & =-\int_{Q} \frac{\partial W}{\partial x_{j}} \times \frac{\partial W}{\partial x_{k}} \times \frac{\partial q_{k}}{\partial x_{j}} d x d t+\frac{1}{2} \int_{Q} \frac{\partial q_{k}}{\partial x_{k}}|\nabla W|^{2} d x d t . \\
& +\frac{1}{2} \int_{\sum} q_{k} \eta_{k}\left|\frac{\partial q_{k}}{\partial x_{k}}\right|^{2} d \Sigma
\end{aligned}
$$

finally since

$$
\int_{Q} h(W) q_{k} \frac{\partial W}{\partial x k} d x d t=\int_{Q} \frac{\partial}{\partial x_{k}} H(W) q_{k}=\int_{Q} H(W) \frac{\partial}{\partial x_{k}} q_{k} .
$$

we have from $(2.22),(2.23),(2.24)$ and $(2.25)$ that the result follows.

REMARK 2.4.- From Lemma 2.3 taking $q=\left(q_{k}\right)$ a field of vectors of class $\left[\mathcal{C}^{\prime}(\bar{\Omega})\right]^{\eta}$, such that

$$
q_{k}=\eta_{k} \text { on } \sum_{j}
$$

and putting:

$$
\mathcal{C}=\sup \left\{\left|q_{k}(x)\right|,\left|\frac{\partial q_{k}}{\partial x_{k}}(x)\right| ; k, j=1, \ldots, n \text { and } \mathbf{x} \in \bar{\Omega}\right\}
$$


we have:

$$
\begin{aligned}
& \left.\frac{1}{2} \int\left|\frac{\partial H}{\partial \eta}\right|^{3} d L \leq 2 n: \Delta \eta\right)_{(0, I)} J(t)+ \\
& +C n T \sup _{\{0, T\}} J(t)+C n \int_{Q}\left|H\left(H^{\circ}\right)\right| d x d t+ \\
& +2 \mathcal{C}(\sup J(t))+\frac{c n}{2}\left|I^{\prime}\right|_{Q}^{2}+C T \sup J(t)
\end{aligned}
$$

From where we have:

$$
\begin{gathered}
\frac{1}{2} \int_{\sum}\left|\frac{\partial W}{\partial \eta}\right| d \sum \leq \mathcal{C}(n+1)(2+T) \sup _{[0, T]} J(t)+ \\
+\mathcal{C} n \frac{1}{2}|V|_{Q}^{2}+\int_{Q}\left|H\left(W^{\prime}\right)\right| d x d t
\end{gathered}
$$

REMARK 2.5.- From (2.21) we have that

$$
W^{\prime \prime}-\Delta W+h(W)+b W=V+b W
$$

mutiplying this expression by $W^{\prime}$ and integrating in $\Omega$ we have:

$$
\frac{d}{d t}\left\{J(t)+\int_{Q} H\left(W^{\prime}\right)+b|W|^{2} d x\right\}=\left(V, W^{\prime}\right)_{\Omega}+b\left(V, W^{\prime}\right)_{\Omega}
$$

where $J(t)=\frac{1}{2}\left\{\left|W^{\prime}(t)\right|^{2}+\|W(t)\|^{2}\right\}$. If we put $\mathcal{C}_{0}=\max \left\{1, b+1, b c^{3}\right\}$ 
(where $\mathrm{c}$ is such that $|\cdot|_{\Omega} \leq\|\cdot\|$ )we obtain, after integrate from 0 to t, that:

$$
J(t)+\int_{Q}\left\{H(W)+b(W)^{2}\right\} d x \leq \frac{1}{2}|V|_{Q}^{2}+2 \mathcal{C}_{0} \mathbf{E}(0)+\mathcal{C}_{0} \int_{0}^{1} J(s) d s
$$

where $\mathrm{E}(t)$ is the energy associate with system (2.21), that is:

$$
\mathbf{E}(t)=J(t)+\int_{\Omega} H(W(x, t)) d x
$$

REMARK 2.6.- Multiplying $(2.21)_{1}$ by $\mathrm{W}$, integrating in $Q$, and applying Green's formuls, we have that:

$$
\begin{gathered}
\int_{Q} W h(W) d x d t=\int_{Q} W V d x d t+\int_{0}^{T}\left|W^{\prime}(t)\right|_{\Omega}^{2} d t- \\
-\int_{0}^{T}\|W(t)\|^{2} d t-\left(W^{\prime}(t), W(t)\right)_{0^{\prime}}^{T}
\end{gathered}
$$

from where we have that:

$$
\int_{Q} W h(W) d x d t \leq \frac{1}{2}|V|_{Q}^{2}+(3 T+2 C) \sup _{\{0, T\}} J(t)
$$

(where $\mathcal{C}$ is such that $|\cdot|_{\Omega} \leq C\|\cdot\|$ ).

Now we are condition to prove the main result of this paper: THEOREM 2.7.- Let $\left(u_{0}, u_{1}, V\right)$ be an elemente of the space 
$H_{0}^{1}(\Omega) \times L^{2}(\Omega) \times L^{2}(Q)$, and let $f$ b a continuous function such that $\left(i\left(n_{0}\right) \in\right.$ $L^{1}(())$. Then there exist a function $u: Q \rightarrow \mathbb{R}$ satisfying.

$$
u \in L^{\infty}\left(0, T: H_{0}^{1}(\Omega)\right) . u^{\prime} \in L^{\infty}\left(0, T: H^{2}(\Omega)\right)
$$

$$
\begin{cases}u^{\prime \prime}-\Delta u+f(u)=V & \text { in } Q \\ u(0)=u_{0}, u^{\prime}(0)=u_{1} & \text { in } \Omega \\ u(x, t)=0 & \text { on } \sum\end{cases}
$$

REMARK 2.8.- We are proving here that, exist one solution satisfying the last. two conditions. We don't know if all solution of (1.1) satisfies this regularity result. This is an open question.

PROOF OF THEOREM 2.7.- Let $\left(\rho_{\mu}\right)_{\mu \in \mathbb{N}}$ be a regularizant sequence on $\mathbb{R}$. That is: $\rho_{\mu} \in \mathcal{C}^{\infty}(\mathbb{R}),. \forall \mu \in \mathbb{N}$ and:

$$
\begin{gathered}
\left.\rho_{\mu}(s) \geq 0 \quad \forall s \in \mathbb{R} \quad \text { and } \operatorname{sopp}\left(\rho_{\mu}\right) \subset\right]-\frac{1}{\mu}, \frac{1}{\mu}[ \\
\int_{\mathbb{R}} \rho_{\mu}(s) d s=1 \quad \forall \mu \in \mathbb{N}
\end{gathered}
$$


Let us denote by $\left(f_{\nu_{\mu}}\right)_{\mu \in \mathbb{N}}$ the sequence of bounded function defined by:

$$
f_{v \mu}=f_{v}{ }^{\circ} \rho_{\mu} \text { for a fixed } \nu \text {. }
$$

Then we have $f_{n m}$ is a $\mathcal{C}^{\infty}$ bounded function we now consider the following aproximated problem.

$$
\begin{cases}u_{\nu \mu}^{\prime \prime}-\Delta u_{\nu \mu}+f\left(u_{\nu \mu}\right)=V & \text { in } \quad Q \\ u_{\nu \mu}(0)=u_{0}, u_{V \mu}^{\prime}=u_{1} & \text { in } \quad \Omega ; \\ u_{\nu \mu}(x, t)=0 & \text { on } \sum\end{cases}
$$

As well known that for every $\left(u_{0}, u_{1}, V\right) \in H_{0}^{1}(\Omega) \times L^{2}(\Omega) \times L^{2}(Q)$ there exist. an unique solution for system (2.33). In order to obtain the existence of a solution for the system (1.3), let us suppose that, $V, u_{0}, u_{1}$ be test function, then we have that:

$$
\begin{gathered}
u_{\nu \mu} \in L^{\infty}\left(0, T ; H_{0}^{1}(\Omega) \eta H^{2}(\Omega)\right) . \\
u_{\nu \mu} \in L^{\infty}\left(0, T ; H_{0}^{1}(\Omega)\right)
\end{gathered}
$$




$$
u_{\nu \mu} \in L^{\infty}\left(0, T ; H^{2}(\Omega)\right)
$$

From Remark (2.4) we have that the normal derivate of $u_{\nu \mu}$, satisfies the following inequality:

$$
\begin{gathered}
\frac{1}{2} \int_{\sum}\left|\frac{\partial u_{\nu \mu}}{\partial \eta}\right|^{2} d \sum \leq \mathcal{C}(n+1)(2+T) \sup J_{\nu \mu}(t)+ \\
+c n\left\{\frac{1}{2}|V|_{Q}^{2}+\int_{Q}\left|G_{\nu \mu}\left(u_{\nu \mu}\right)\right|\right\} d x d t .
\end{gathered}
$$

Where by $J_{\nu \mu}(t)$ we are denoting the quadratic term associated to system (2.36), that is:

$$
J_{\nu \mu}(t)=\frac{1}{2}\left|u_{\nu \mu}(t)\right|_{\Omega}^{2}+\frac{1}{2}\left\|u_{\nu \mu}(t)\right\|^{2}
$$

By Remarks 2.5, we have that,

$$
\begin{aligned}
J_{\nu \mu}(t) & +\int_{\Omega} G_{\nu \mu}\left(u_{V \mu}\right)+b\left|u_{\nu \mu}\right|^{2} d x \leq \frac{1}{2}|V|_{Q}^{2}+ \\
& +2 \mathcal{C}_{Q} E_{\nu \mu}(0)+\mathcal{C}_{0} \int_{0}^{T} J_{\nu \mu}(s) d s .
\end{aligned}
$$

and since $\mathrm{b}$ is a positive number, and $G_{\nu \mu}$ is uniformly bounded for all $\mu \in \mathbb{N}$, and 
a fixed $v$, we have by Gronwall inequality that there exist a constand $\mathcal{C}_{\nu}$ such us:

$$
J_{\nu \mu}(t)=\frac{1}{2}\left|u_{\nu \mu}^{\prime}(t)\right|_{\Omega}^{2}+\frac{1}{2}\left\|u_{\nu \mu}(t)\right\|^{2} \leq \mathcal{C}_{\nu}
$$

where $E_{\nu \mu}(t)$ is the energy associated to system (2.36) with non quadratic term $G_{\nu \mu}\left(u_{\nu \mu}\right)$

Finally from Remark 2.6 we obtain that:

$$
\begin{gathered}
\int_{Q} u_{\nu \mu} f_{V \mu}\left(u_{\nu \mu}\right) d x \leq+ \\
+\frac{1}{2}|V|_{Q}^{2}+(3 T+2 \mathcal{C}) \sup _{\{0, T\}} J_{V \mu}(t) .
\end{gathered}
$$

Relation (2.41), (2.42), and (2.43) are valid when $V, u_{0}$ and $u_{1}$ are test function. If we take a sequence of test function $\left(V_{m}, u_{0 m}, u_{1 m}\right)$ satisfying,

$$
\left(V_{m}, u_{0 m}, u_{1 m}\right) \rightarrow\left(V, u_{0}, u_{1}\right) \text { strongly } \quad \text { in } L^{2}(Q) \times H_{0}^{1}(\Omega) \times L^{2}(\Omega)
$$

certainly we have that the corresponding solutions $u_{\nu u m}$ converge to $u_{\nu u}$ solution of system (2.36), when the datas $V, u_{0}$ and $u_{1}$ are $L^{2}(Q), H_{0}^{1}(\Omega)$ and $L^{2}(\Omega)$ 
respectively. Moreover we have:

$$
\begin{aligned}
& u_{\nu u m} \rightarrow u_{\nu u} \text { strongly in } L^{\infty}\left(O, T ; H_{0}^{1}(\Omega)\right) . \\
& u_{\nu u m} \rightarrow u_{\nu u} \text { strongly in } L^{\infty}\left(O, T ; L^{2}(\Omega)\right)
\end{aligned}
$$

From (2.44) and (2.45) we conclude that relations $(2.40),(2.41),(2.42)$ and (2.43) are valid when $\left(V, u_{0}, u_{1}\right)$ belongs to $L^{2}(Q) \times H_{0}^{1}(\Omega) \times L^{2}(\Omega)$ and $u_{\nu u}$ is solution of (3.36).

On the other hand, by (2.42) we obtain that there exists a subsequence of $\left(u_{\nu \mu}\right)_{\mu \in \mathrm{N}}$ satisfying:

$$
\begin{aligned}
& u_{\nu \mu}^{\prime} \rightarrow u_{\nu} \text { weak star in } L^{\infty}\left(O, T ; H_{0}^{1}(\Omega)\right) \\
& u_{\nu \mu}^{\prime} \rightarrow u_{\nu}^{\prime} \text { weak star in } L^{\infty}\left(O, T ; L^{2}(\Omega)\right)
\end{aligned}
$$

from (2.46) and (2.47) we have that there exist another subsequence (that we still denoting in the same way) such that,

$$
u_{\nu \mu} \rightarrow u_{\nu} \text { strongly in } L^{2}(Q) .
$$




$$
\begin{gathered}
u_{\nu \mu} \rightarrow u_{\nu} \text { a. e. in } Q . \\
f_{\nu \mu}\left(u_{\nu \mu}\right) \rightarrow f_{v}\left(u_{v}\right) \text { a. e. in } Q . \\
G_{\nu \mu}\left(u_{\nu \mu}\right) \rightarrow G_{v}\left(u_{v}\right) \text { a. e. in } Q .
\end{gathered}
$$

Since $f_{\nu \mu}$ is bounded for all $\mu \in \mathbb{N}$ ( $v$ fixed), then $G_{\nu \mu}$ is a Lipschitz's in $\mathbf{R}$, then by Lebesgue dominated convergence theorem we conchude that:

$$
\begin{gathered}
f_{\nu \mu}\left(u_{\mu \mu}\right) \rightarrow f_{\nu}\left(u_{\nu}\right) \text { strongly in } L^{2}(Q) . \\
G_{\nu \mu}\left(u_{\nu \mu}\right) \rightarrow G_{\nu}\left(u_{\nu}\right) \text { strongly in } L^{2}(Q) .
\end{gathered}
$$

Now, from (2.48) and (2.52) we obtain:

$$
\begin{aligned}
& u_{\nu \mu} \rightarrow u_{\nu} \text { strongly in } L^{\infty}\left(O, T ; H_{0}^{1}(\Omega)\right) . \\
& u_{\nu \mu}^{\prime} \rightarrow u_{\nu}^{\prime} \text { strongly in } L^{\infty}\left(O, T ; L^{2}(\Omega)\right) .
\end{aligned}
$$

Then by $(2.40),(2.42)$ and $(2.53)$ we obtain that there exist a subsequence of $\frac{\partial u}{\partial \eta} \nu \mu$, which we still denote in the same way and a element $\mathcal{X}$ in $L^{2}(\Sigma)$ such 
that:

$$
\frac{\partial u_{\nu \mu}}{\partial \eta} \rightarrow \mathcal{X} \nu \text { weak in } L^{2}\left(\sum\right)
$$

But since:

$$
\frac{\partial u_{\nu \mu}}{\partial \eta} \rightarrow \frac{\partial u_{\nu}}{\partial \eta} \text { weak in } H^{-1}\left(O, T ; H^{1 / 2}(\Gamma)\right)
$$

we conclude that $\mathcal{X}_{\nu}=\frac{\partial u_{\nu}}{\partial \eta}$. From (2.54) and (2.55) we have in particular that:

$$
\begin{aligned}
& J_{\nu \mu}(t) \rightarrow J_{\nu}(t) \text { uniformly on }[O, T] \\
& E_{\nu \mu}(t) \rightarrow E_{\nu}(t) \text { uniformly on }[O, T]
\end{aligned}
$$

Then form $(2.40),(2.53)$ and (2.57) we obtain that:

$$
\begin{gathered}
\frac{1}{2} \int_{\sum}\left|\frac{\partial u_{\nu}}{\partial \eta}\right|^{2} d \sum \leq \mathcal{C}(n+1)(2+T) \sup _{\{O, T\}} J_{\nu}(t)+ \\
+\mathcal{C} n\left\{\frac{1}{2}|V|_{Q}^{2}+\int_{Q}\left|G_{\nu}\left(u_{\nu}\right)\right|\right\} d x d t .
\end{gathered}
$$

Now by $(2.41),(2.43),(2.48),(2.53),(2.57)$ and $(2.58)$ we obtain:

$$
\begin{gathered}
J_{\nu}(t)+\int_{\Omega}\left\{G_{\nu}\left(u_{\nu}\right)+b\left|u_{\nu}\right|^{2}\right\} \leq \\
\leq \frac{1}{2}|V|_{Q}^{2}+2 \mathcal{C}_{0} E_{\nu}(0)+\mathcal{C}_{0} \int_{0}^{t} J_{\nu}(s) d s .
\end{gathered}
$$




$$
\int u_{\nu} f_{\nu}\left(u_{\nu}\right) d x d t \leq \frac{1}{2}|V|_{Q}^{2}+(3 T+2 \mathcal{C}) \sup _{[O, T]} J_{\nu}(t)
$$

From (2.10) and (2.59) taking $b$ such that $G_{\nu}\left(u_{\nu}\right)+b\left|u_{\nu}\right|^{2}$ be positive, we obtain:

$$
J_{\nu}(t) \leq \frac{1}{2}|V|_{Q}^{2}+2 \mathcal{C}_{0} E_{\nu}(0)+\mathcal{C}_{0} \int_{\Omega}^{t} J_{\nu}(s) d s
$$

Now by Gronwal's inequality we obtain that:

$$
J_{\nu}(t) \leq\left\{\frac{1}{2}|V|_{Q}^{2}+2 \mathcal{C}_{0} E_{\nu}(0)\right\} \mathrm{e}^{C_{0} T}, \forall t \in[O, T]
$$

Since,

$$
E_{\nu}(0)=\frac{1}{2}\left\{\left\|u_{0}\right\|^{2}+\left|u_{1}\right|_{\Omega}^{2}\right\}+\int_{\Sigma} G_{\nu}\left(u_{0}\right) d x,
$$

we conclude from (2.12) and the bypothesis of Theorem 2.6, that the second member of (2.63) is bounded by a constand $\mathcal{C}_{1}>0$, independing of $\nu$, then from (2.63) we have:

$$
\sup _{\mid O, T]} J_{\nu}(t) \leq\left\{\frac{1}{2}|V|_{Q}^{2}+2 \mathcal{C}_{0} E_{\nu}(0)\right\} \mathrm{e}^{C_{0} T} \leq \mathcal{C}_{1}
$$

Then we have that there exist a subsequence of $\left(u_{\eta}\right)_{\eta \mathrm{N}}$, that we still 
denote on the same way, and a element. $u \in L^{\infty}\left(O, T ; H_{0}^{1}(\Omega)\right)$ such that $u^{\prime} \in L^{\infty}\left(O, T ; L^{2}(\Omega)\right)$, satisfying:

$$
\begin{gathered}
u \in L^{\infty}\left(O, T ; H_{0}^{1}(\Omega)\right) \text { such that } u^{\prime} \in L^{\infty}\left(O, T ; L^{2}(\Omega)\right), \\
u_{\nu}^{\prime} \rightarrow u^{\prime} \text { weak st.ur in } L^{\infty}\left(O, T ; L^{2}(\Omega)\right) .
\end{gathered}
$$

By (2.61) and (2.63) we obtain that:

$$
\int_{Q} u_{\nu} f_{\nu}\left(u_{\nu}\right) d x \leq 3 \mathcal{C}_{2} \mathrm{e}^{C_{0} T}\left\{|V|_{Q}^{2}+E_{\nu}(0)\right\} \leq \mathcal{C}_{3}
$$

where $\mathcal{C}_{2}=\max \left\{n, T, \mathcal{C}, \mathcal{C}_{0}\right\}$. But from (2.9) we obtain that $\left|u_{\nu} f_{\nu}\left(u_{\nu}\right)\right| \leq$ $u_{\nu} f_{\nu}\left(u_{\nu}\right)+2 \mathcal{C}_{0}\left(u_{\nu}^{2}+1\right)$, from where we have:

$$
\int_{Q}\left|u_{\nu} f_{\nu}\left(u_{\nu}\right)\right| d x \leq \mathcal{C}_{3}+4 \mathcal{C}_{0} \times \mathcal{C}_{1} \operatorname{med}(Q)=\mathcal{C}_{4}
$$

Then by (2.68) and from Theorem 1.1 of W.A. Strauss [4] we have that:

$$
f_{\nu}\left(u_{\nu}\right) \rightarrow f_{(u)} \text { strongly in } L^{1}(Q) .
$$

Then we conclude that $u$ is a solution of problem (1.1). Finally from (2.59) 
and (2.63) we have that there exist. a constant $\mathcal{C}_{5}$ (independing of $\nu$ ) such that:

$$
\int_{\sum}\left|\frac{\partial u_{\nu}}{\partial \eta}\right| d \sum \leq \mathcal{C}_{s}\left\{|V|_{Q}^{2}+E_{\nu}(0)+\int_{Q}\left|G_{\nu}\left(u_{v}\right)\right| d x d t\right\} .
$$

But from $(2.60)$ and $(2.64)$ we have that exist a constant $\mathcal{C}_{6}$ such that,

$$
\int_{Q} G_{\nu}\left(u_{\nu}\right) d x \leq \mathcal{C}_{8}\left\{|V|_{Q}^{2}+E_{\nu}(0)\right\}
$$

Now from $(2.10)$ we have that $G_{\nu}(t) \leq-\mathcal{C}_{0}\left(t^{2}+2|t|\right)$, from where we conchide that $\left|G_{\nu}(t) \leq G_{\nu}(t)\right|+2 \mathcal{C}_{0}\left(t^{2}+2|t|\right)$ taking $t=u_{n}$ we have after to integrate in $Q$, that:

$$
\int_{Q} G_{\nu}\left(u_{\nu}\right) d x d t \leq \int_{Q} G_{\nu}\left(u_{\nu}\right) d x d t+2 \mathcal{C}_{0} \int_{Q} u_{\nu}^{2}+2\left|u_{\nu}\right| d x d t
$$

On the other hand, there exist a constant $\mathcal{C}_{7}$ such that:

$$
\int_{Q}\left|u_{\nu}\right|+2\left|u_{\nu}\right| d x d t \leq \mathcal{C}_{7} \sup _{\{0, T\}} J_{\nu}(t)
$$

From $(2.70),(2.71),(2.72)$ and $(2.73)$ we obtain another constant, say $\mathcal{C}_{8}$ such 
that:

$$
\int_{\Sigma}\left|\frac{\partial u_{\nu}}{\partial \eta}\right| d \Sigma \leq \mathcal{C}_{8}\left\{|V|_{Q}^{2}+E_{\nu}(0)\right\}
$$

Since the second member of $(2.74)$ is bounded we obtain a subsecuence of,

$$
\left(\frac{\partial u_{\nu}}{\partial \eta}\right)_{\nu \in \mathbb{N}}
$$

and a element $\mathcal{X}$ in $L^{2}(\Sigma)$ such that:

$$
\frac{\partial u_{\nu}}{\partial \eta} \rightarrow \mathcal{X} \text { weak in } L^{2}(\Sigma)
$$

But,

$$
\frac{\partial u_{\nu}}{\partial \eta} \rightarrow \frac{\partial u_{\nu}}{\partial \eta} \text { in } H^{-1}\left(0, T ; W^{-1 / p-2, p^{\prime}}(\Gamma)\right)
$$

Where $p>\frac{n}{2}$, then we have that $\mathcal{X}=\frac{\partial u}{\partial \eta}$, and letting $\nu \rightarrow \infty$ in (2.74) we have,

$$
\int_{\sum}\left|\frac{\partial u}{\partial \eta}\right|^{2} d \sum \leq \mathcal{C}_{8}\left\{|V|_{Q}^{2}+E(0)\right\} \quad \text { Q.E.D. }
$$

\section{REFERENCES}

1.- J. L. Lions - Hidden regularity in some nonlinear hyperbolic equations. Matemática Aplicada e Computacional, v.6, n.1, pp. 7-15, (1987). 
2.- J. L. Lions - Quelques méthodes de résolution des problemes aux limites nonlineares, Paris. Dunod, (1969).

3.- J. L. Lions - Controlabilité exate de systemes distribues, vol. 1, College de France, Departament de Mathématiques, n. 3,, Rue d'Ulm 75005, Paris, France.

4.- W. A. Strauss - On weak solution of semilinear hyperbolic equations, $A n$, Acad. Brasil. Ciencias, vol. 42, n,4, pp. 645 - 651, (1970). 